\title{
Comparison of a Subtotal Colectomy With Self-Expandable Metallic Stent in the Management of Patients With Obstructive Left Colon Cancer
}

\author{
Byung Chun Kim \\ Department of Surgery, Kangnam Sacred Heart Hospital, Hallym University College of Medicine, Seoul, Korea
}

See Article on Page 215-220

Colorectal obstruction caused by colorectal cancer has been reported to occur in $7 \%-29 \%$ of all patients with colorectal cancers $[1,2]$, with left-sided colonic obstruction being more common than right-sided colonic obstruction [3]. Massive colonic distension, localized or generalized peritonitis, fluid and electrolyte imbalance, colonic wall necrosis, and perforation may develop as a result of colorectal obstruction. If a colorectal obstruction does occur, urgent decompression, surgical or medical, is needed. Obstruction or perforation of a colorectal carcinoma in an emergency situation may be associated with worse oncological outcomes and a higher incidence of local spread and metastatic disease [4]. Emergency operations are associated with mortality in $15 \%-34 \%$ of patients and morbidity in 32\%-64\% [2, 3].

Surgical management of an acute malignant left-sided colorectal obstruction remains controversial. In emergency situations, several operative strategies are available to the surgeon, including 2-stage surgery involving primary resection with a colostomy or proximal colostomy followed by resection and 1-stage surgery involving a primary resection with anastomosis. One-stage procedures seem to be superior to 2- or 3-stage procedures in terms of morbidity and mortality. Although few studies regarding 1-stage procedures for the treatment of patients with malignant left-sided colorectal obstruction have been published, recent guidelines recommend either a segmental colectomy and primary anastomosis in cases involving rectal anastomosis or preexisting continence

Correspondence to: Byung Chun Kim, M.D.

Department of Surgery, Kangnam Sacred Heart Hospital, Hallym University College of Medicine, 1 Singil-ro, Yeongdeungpo-gu, Seoul 07441, Korea

Tel: +82-2-829-5130, Fax: +82-2-849-4469,

E-mail: bckimgs@hallym.or.kr

(c) 2016 The Korean Society of Coloproctology

This is an open-access article distributed under the terms of the Creative Commons Attribution NonCommercial License (http://creativecommons.org/licenses/by-nc/4.0) which permits unrestricted noncommercial use, distribution, and reproduction in any medium, provided the original work is properly cited. disturbance or a subtotal colectomy and primary anastomosis in cases involving cecal wall necrosis, colon perforation or serosal tear, or synchronous colonic tumor [5]. In 2- or 3-stage procedures, restoration of the stoma requires a second major operation due to the patient's old age, poor general condition and combined disease. Restoration of the stoma is performed in only $60 \%$ of cases, and its morbidity rate is about $40 \%$ [6]. Patients with a colostomy have poorer health-related quality of life than do patients without a colostomy.

A self-expandable metallic stent (SEMS), as a bridge to surgery, might be a useful option for 1-stage surgery without a colostomy. Its clinical success rate is $84 \%$ to $94 \%$, the stent migration rate is $12 \%$, the reobstruction rate is $7 \%$, and the clinical stent perforation rate is $4 \%-6.9 \%[7,8]$. The stent-procedure-related mortality in patients with colonic obstruction is reported to be less than $1 \%$ [8]. Placement of the SEMS can decompress the colon, enabling conversion of an emergency surgery to an elective one with successful primary anastomosis without a colostomy. The SEMS procedure allows the surgeon to perform elective laparoscopic surgery on patients with an acute malignant left-sided colorectal obstruction. Laparoscopic surgery for such patients has many advantages, including a reduced number of sequential operations, a reduction in stoma requirements, and a reduction in overall morbidity and mortality. Nevertheless, we have to admit that the SEMS has an adverse effect on the 5-year overall and disease-free survival rates [9].

In this study, 94 consecutive patients with left colon cancer obstruction who received an emergency subtotal colectomy or SEMS insertion as elective surgery were enrolled. A subtotal colectomy was attempted in 24 patients, and preoperative SEMS insertion was attempted in 70 patients. Laparoscopic surgery was performed more frequently on the patients in the SEMS group (62 of $70,88.6 \%$ ) compared with patients in the subtotal colectomy group ( 4 of $24,16.7 \%$ ). Total hospital stay was shorter in the subtotal colectomy group. The median number of bowel movements in the subtotal colectomy group was twice per day at postoperative 3-6 months. A subtotal colectomy for the treatment of pa- 
tients with obstructive left colon cancer is a clinically and oncologically safer 1-stage surgical strategy compared to SEMS insertion as a bridge to surgery. A subtotal colectomy can treat synchronous tumors in the proximal colon, reduce total hospital stays, and avoid oncologic deterioration from colonic stenting, as bowel function is preserved. Preoperative SEMS insertion should be considered only for patients with rectal or rectosigmoid junction cancer and patients for whom minimally invasive surgery is planned [10].

Obviously, further safety and efficacy evaluations of SEMS placement as a bridge to surgery are needed to assess its feasibility and expected benefits compared with a subtotal colectomy. Further studies are necessary to compare the oncological outcomes of the two strategies.

\section{CONFLICT OF INTEREST}

No potential conflict of interest relevant to this article was reported.

\section{REFERENCES}

1. Deans GT, Krukowski ZH, Irwin ST. Malignant obstruction of the left colon. Br J Surg. 1994;81:1270-6.

2. Smothers L, Hynan L, Fleming J, Turnage R, Simmang C, Anthony T. Emergency surgery for colon carcinoma. Dis Colon Rectum 2003;46:24-30.
3. Tekkis PP, Kinsman R, Thompson MR, Stamatakis JD; Association of Coloproctology of Great Britain, Ireland. The Association of Coloproctology of Great Britain and Ireland study of large bowel obstruction caused by colorectal cancer. Ann Surg 2004; 240:76-81.

4. McArdle CS, Hole DJ. Emergency presentation of colorectal cancer is associated with poor 5-year survival. Br J Surg 2004;91:605-9.

5. Finan PJ, Campbell S, Verma R, MacFie J, Gatt M, Parker MC, et al. The management of malignant large bowel obstruction: ACPGBI position statement. Colorectal Dis 2007;9 Suppl 4:1-17.

6. Pearce NW, Scott SD, Karran SJ. Timing and method of reversal of Hartmann's procedure. Br J Surg 1992;79:839-41.

7. Watt AM, Faragher IG, Griffin TT, Rieger NA, Maddern GJ. Selfexpanding metallic stents for relieving malignant colorectal obstruction: a systematic review. Ann Surg 2007;246:24-30.

8. Sebastian S, Johnston S, Geoghegan T, Torreggiani W, Buckley M. Pooled analysis of the efficacy and safety of self-expanding metal stenting in malignant colorectal obstruction. Am J Gastroenterol 2004;99:2051-7.

9. Kim JS, Hur H, Min BS, Sohn SK, Cho CH, Kim NK. Oncologic outcomes of self-expanding metallic stent insertion as a bridge to surgery in the management of left-sided colon cancer obstruction: comparison with nonobstructing elective surgery. World J Surg 2009;33:1281-6.

10. Min CK, Kim HO, Lee D, Jung KU, Lee SR, Kim H, et al. Obstructive left colon cancer should be managed by using a subtotal colectomy instead of colonic stenting. Ann Coloproctol 2016;32:215-20. 